\title{
SPECTRAL DISTANCES IN SOME SETS OF GRAPHS
}

\author{
IRENA M. JOVANOVIĆ
}

\begin{abstract}
Some of the spectral distance related parameters (cospectrality, spectral eccentricity, and spectral diameter with respect to an arbitrary graph matrix) are determined in one particular set of graphs. According to these results, the spectral distances connected with the adjacency matrix and the corresponding distance related parameters are computed in some sets of trees. Examples are provided of graphs whose spectral distances related to the adjacency matrix, the Laplacian and the signless Laplacian matrix are mutually equal. The conjecture related to the spectral diameter of the set of connected regular graphs with respect to the adjacency matrix is disproved using graph energy.
\end{abstract}

\section{INTRODUCTION}

The problems related to the Manhattan spectral distance of graphs were posed by Richard Brualdi at the Aveiro Workshop on Graph Spectra 2006 (see [14]).

Let $G_{i}$ and $G_{j}$ be two non-isomorphic graphs on $n$ vertices whose spectra with respect to some graph matrix $M$ are $m_{1}\left(G_{k}\right) \geq m_{2}\left(G_{k}\right) \geq \cdots \geq m_{n}\left(G_{k}\right), k=i, j$. The $M$-spectral distance $\sigma_{M}\left(G_{i}, G_{j}\right)$ between $G_{i}$ and $G_{j}$ is the Manhattan distance between their $M$-spectra:

$$
\sigma_{M}\left(G_{i}, G_{j}\right)=\sum_{s=1}^{n}\left|m_{s}\left(G_{i}\right)-m_{s}\left(G_{j}\right)\right| .
$$

In this paper, the spectral distances are considered regarding the adjacency matrix $A$, the Laplacian $L=D-A$ and the signless Laplacian matrix $Q=D+A$, where $D$ is the diagonal matrix of vertex degrees. The characteristic polynomial $P_{G_{i}}(x)=\operatorname{det}(x I-A)$ of $G_{i}$ is the characteristic polynomial of its adjacency ma$\operatorname{trix} A$, while the eigenvalues of $G_{i}$ connected with the adjacency matrix $A$ are denoted by $\lambda_{1}\left(G_{i}\right) \geq \lambda_{2}\left(G_{i}\right) \geq \cdots \geq \lambda_{n}\left(G_{i}\right)$.

Let $I$ be a family of indices, and $\mathcal{G}=\left\{G_{i}: i \in I\right\}$ a set of non-isomorphic graphs of order $n$. The $M$-cospectrality of $G_{i} \in \mathcal{G}$ is $\operatorname{cs}_{\mathcal{G}}^{M}\left(G_{i}\right)=\min \left\{\sigma_{M}\left(G_{i}, G_{j}\right)\right.$ : $\left.G_{j} \in \mathcal{G}, i \neq j\right\}$, while the $M$-cospectrality measure of the set $\mathcal{G}$ is $\operatorname{cs}^{M}(\mathcal{G})=$

2020 Mathematics Subject Classification. 05C50.

Key words and phrases. Adjacency matrix; graph spectrum; spectral distance; graph energy.

This work is supported by the Serbian Ministry of Education, Science and Technological Development, through the Mathematical Institute of the Serbian Academy of Sciences and Arts. 
$\max \left\{\operatorname{cs}_{\mathcal{G}}^{M}\left(G_{i}\right): G_{i} \in \mathcal{G}\right\}$. In [9], the $M$-spectral eccentricity of $G_{i}, \operatorname{secc}_{\mathcal{G}}^{M}\left(G_{i}\right)=$ $\max \left\{\sigma_{M}\left(G_{i}, G_{j}\right): G_{j} \in \mathcal{G}, i \neq j\right\}$, and the $M$-spectral diameter of $\mathcal{G}, \operatorname{sdiam}^{M}(\mathcal{G})=$ $\max \left\{\operatorname{secc}_{\mathcal{G}}^{M}\left(G_{i}\right): G_{i} \in \mathcal{G}\right\}$, are also introduced.

Two problems, originally presented in [14, are: Investigate $\operatorname{cs}_{\mathcal{G}}^{M}\left(G_{i}\right)$ for special classes of graphs $\mathcal{G}$, and search for a good upper bound on $\operatorname{cs}^{M}(\mathcal{G})$. Some results related to these problems can be found in [1, [9], [10] and [8, while in [2] the problem related to $\operatorname{cs}^{M}(\mathcal{G})$, regarding the spectral distance $\sigma_{A}\left(G_{i}, G_{j}\right)=$ $\sum_{s=1}^{n}\left(\lambda_{s}\left(G_{i}\right)-\lambda_{s}\left(G_{j}\right)\right)^{2}$ was completely solved. Namely, the authors proved that $\operatorname{cs}^{A}(\mathcal{G})=2$, for $n \geq 2$, whenever $\operatorname{cs}^{A}(\mathcal{G})$ is computed with respect to any $\ell^{p}$-norm, for $1 \leq p<\infty$, and $\operatorname{cs}^{A}(\mathcal{G})=1$, with respect to the $\ell^{\infty}$-norm.

Further, in [9] the following is conjectured:

Conjecture 1.1. Let $R_{1}$ and $R_{2}$ be the graphs having the maximum A-spectral distance among the connected regular graphs of order $n$. Then, one of them is the complete graph on $n$ vertices.

In this paper, the parameters of spectral distances, namely the $M$-cospectrality, the $M$-spectral eccentricity and the $M$-spectral diameter, will be computed in some particular sets of graphs, and Conjecture 1.1 will be disproved.

The notation common for spectral graph theory is used in the paper. In that way, $P_{n}$ is the path on $n$ vertices, $C_{n}$ is the cycle of order $n, K_{n}$ is the complete graph on $n$ vertices, while $K_{1, n}$ is the star of order $n+1$. For the complete multipartite graph on $p$ parts and $k$ vertices in each of them, i.e. for the complete $p$-partite graph, the expression $K_{\underbrace{k, k, \ldots, k}_{p}}$ or $K_{p \times k}$ is utilized.

The complement of $G$ is denoted by $\bar{G}$. The graph $G_{i} \cup G_{j}$ means the disjoint union of the graphs $G_{i}$ and $G_{j}$, while the disjoint union of $k$ copies of the same graph $G_{i}$ is labeled by $k G_{i}$. The join $G_{i} \nabla G_{j}$ of disjoint graphs $G_{i}$ and $G_{j}$ is the graph obtained from $G_{i} \cup G_{j}$ by joining each vertex of $G_{i}$ to each vertex of $G_{j}$. The following result will be used in some proofs in Section 3.

Theorem 1.2 ([5, Theorem 2.1.8]). If $G_{i}$ is a $r_{i}$-regular graph with $n_{i}$ vertices, and $G_{j}$ is a $r_{j}$-regular graph with $n_{j}$ vertices, then the characteristic polynomial of the join $G_{i} \nabla G_{j}$ is given by

$$
P_{G_{i} \nabla G_{j}}(x)=\frac{P_{G_{i}}(x) P_{G_{j}}(x)}{\left(x-r_{i}\right)\left(x-r_{j}\right)}\left(\left(x-r_{i}\right)\left(x-r_{j}\right)-n_{i} n_{j}\right) .
$$

The coalescence $G_{i} \cdot G_{j}$ of two graphs, $G_{i}$ and $G_{j}$, is a graph obtained from $G_{i} \cup G_{j}$ by identifying a vertex $u$ of $G_{i}$ with a vertex $v$ of $G_{j}$. To prove some of the statements in Sections 2 and 3 of the paper, it is necessary to recall the following statement related to the graph operation of coalescence.

Theorem 1.3 ([5, Theorem 2.2.3]). Let $G_{i} \cdot G_{j}$ be the coalescence in which the vertex $u$ of $G_{i}$ is identified with the vertex $v$ of $G_{j}$. Then

$$
P_{G_{i} \cdot G_{j}}(x)=P_{G_{i}}(x) P_{G_{j}-v}(x)+P_{G_{i}-u}(x) P_{G_{j}}(x)-x P_{G_{i}-u}(x) P_{G_{j}-v}(x) .
$$


The energy $E\left(G_{i}\right)$ of the graph $G_{i}$ with respect to the adjacency matrix is defined as $E\left(G_{i}\right)=\sum_{s=1}^{n}\left|\lambda_{s}\left(G_{i}\right)\right|$.

For the remaining notation and terminology, the reader is referred to [4] or [5].

The paper is organized as follows: in Section 2, some of the parameters of the $M$-spectral distances (the $M$-cospectrality, the $M$-spectral eccentricity, and the $M$-spectral diameter) are determined in one particular set of graphs. According to these results, the $A$-spectral distance related parameters in some sets of trees are computed. In Section 3, some examples of graphs whose $A$-, $L$ - and $Q$-spectral distances are mutually equal are provided, while in Section 4, Conjecture 1.1 is disproved using graph energy.

\section{Spectral Distances in some sets of trees}

The triangular inequality implies the following result.

Proposition 2.1. Let $\mathcal{G}=\left\{G_{1}, G_{2}, \ldots, G_{f(n)}\right\}$ be the set of non-isomorphic graphs on $n$ vertices, and let $m_{1}\left(G_{k}\right) \geq m_{2}\left(G_{k}\right) \geq \cdots \geq m_{n}\left(G_{k}\right), k=1,2, \ldots, f(n)$, be the $M$-spectra of these graphs with respect to some graph matrix $M$. Then

(1) $\sigma_{M}\left(G_{i}, G_{j}\right) \leq \sigma_{M}\left(G_{i}, G_{k}\right)+\sigma_{M}\left(G_{k}, G_{j}\right)$, for any three $G_{i}, G_{j}, G_{k}$ from $\mathcal{G}$.

(2) $\sigma_{M}\left(G_{1}, G_{f(n)}\right) \leq \sum_{s=1}^{f(n)-1} \sigma_{M}\left(G_{s}, G_{s+1}\right)$.

It is obvious that the equality in (1) in Proposition 2.1 is attained if the graphs $G_{i}, G_{j}$ and $G_{k}$ are mutually cospectral, or if $G_{k}$ is cospectral with one of the graphs $G_{i}$ or $G_{j}$. One more case in which the equality in (1) in Proposition 2.1 is attained is given by the next proposition.

Proposition 2.2. Let $G_{1}, G_{2}, \ldots, G_{f(n)}$ be the sequence of non-isomorphic graphs of order $n$ such that $m_{s}\left(G_{1}\right) \geq m_{s}\left(G_{2}\right) \geq \cdots \geq m_{s}\left(G_{f(n)}\right)$ or $m_{s}\left(G_{1}\right) \leq m_{s}\left(G_{2}\right) \leq$ $\cdots \leq m_{s}\left(G_{f(n)}\right)$, for each $s=1,2, \ldots, n$. Then

$$
\sigma_{M}\left(G_{i}, G_{j}\right)+\sigma_{M}\left(G_{j}, G_{k}\right)=\sigma_{M}\left(G_{i}, G_{k}\right), \quad \text { for } 1 \leq i<j<k \leq f(n) .
$$

Proof. Since $\left|m_{s}\left(G_{i}\right)-m_{s}\left(G_{j}\right)\right|+\left|m_{s}\left(G_{j}\right)-m_{s}\left(G_{k}\right)\right|=\left|m_{s}\left(G_{i}\right)-m_{s}\left(G_{k}\right)\right|$, for each $s=1,2, \ldots, n$, the proof follows.

Remark 2.3. The examples of graphs which are presented in Proposition 2.2 and their $A$-spectral distances are given by Theorem 2.5. Theorem 2.12, and Proposition 3.6

The spectral distance related parameters on the set of graphs of equal order, regarding an arbitrary graph matrix, whose spectral distances satisfy the equality stated in Proposition 2.2 have been computed by the following proposition.

Proposition 2.4. Let $\mathcal{G}=\left\{G_{1}, G_{2}, \ldots, G_{f(n)}\right\}$ be the set of non-isomorphic graphs of order $n$ and let $M$ be an arbitrary graph matrix. If the $M$-spectral distances of graphs from the set $\mathcal{G}$ satisfy (2.1), then the parameters of $M$-spectral distances on the set $\mathcal{G}$ are: 
(1) $\operatorname{cs}_{\mathcal{G}}^{M}\left(G_{i}\right)=\min \left\{\sigma_{M}\left(G_{i}, G_{i+1}\right), \sigma_{M}\left(G_{i-1}, G_{i}\right)\right\}$, for $1<i<f(n)$, and in particular

$$
\begin{aligned}
\operatorname{cs}_{\mathcal{G}}^{M}\left(G_{1}\right) & =\sigma_{M}\left(G_{1}, G_{2}\right), \\
\operatorname{cs}_{\mathcal{G}}^{M}\left(G_{f(n)}\right) & =\sigma_{M}\left(G_{f(n)-1}, G_{f(n)}\right) ;
\end{aligned}
$$

(2) $\operatorname{secc}_{\mathcal{G}}^{M}\left(G_{i}\right)=\max \left\{\sigma_{M}\left(G_{i}, G_{f(n)}\right), \sigma_{M}\left(G_{1}, G_{i}\right)\right\}$, for $1<i<f(n)$, and in particular

$$
\operatorname{secc}_{\mathcal{G}}^{M}\left(G_{1}\right)=\operatorname{secc}_{\mathcal{G}}^{M}\left(G_{f(n)}\right)=\sigma_{M}\left(G_{1}, G_{f(n)}\right) ;
$$

(3) $\operatorname{sdiam}^{M}(\mathcal{G})=\sigma_{M}\left(G_{1}, G_{f(n)}\right)$.

Proof. For fixed $1 \leq i \leq f(n)-2$ and for each $i+1 \leq j \leq f(n)-1$, from (2.1) one can get:

$$
\sigma_{M}\left(G_{i}, G_{i+1}\right) \leq \sigma_{M}\left(G_{i}, G_{i+2}\right) \leq \sigma_{M}\left(G_{i}, G_{i+3}\right) \leq \cdots \leq \sigma_{M}\left(G_{i}, G_{f(n)}\right),
$$
i.e.

$$
\min _{i+1 \leq j \leq f(n)} \sigma_{M}\left(G_{i}, G_{j}\right)=\sigma_{M}\left(G_{i}, G_{i+1}\right)
$$

and

$$
\max _{i+1 \leq j \leq f(n)} \sigma_{M}\left(G_{i}, G_{j}\right)=\sigma_{M}\left(G_{i}, G_{f(n)}\right) .
$$

For fixed $2 \leq i \leq f(n)-1$ and for each $1<j \leq i-1$, one finds $\sigma_{M}\left(G_{1}, G_{i}\right) \geq$ $\sigma_{M}\left(G_{j}, G_{i}\right)$. Since $\sigma_{M}\left(G_{i}, G_{f(n)}\right) \geq \sigma_{M}\left(G_{i+1}, G_{f(n)}\right)$, for $1 \leq i \leq f(n)-2$, one gets

$$
\sigma_{M}\left(G_{1}, G_{f(n)}\right) \geq \sigma_{M}\left(G_{2}, G_{f(n)}\right) \geq \sigma_{M}\left(G_{3}, G_{f(n)}\right) \geq \cdots \geq \sigma_{M}\left(G_{f(n)-1}, G_{f(n)}\right) .
$$

Now, the spectral eccentricities and the spectral diameter can be determined.

For fixed $3 \leq j \leq f(n)$ and for each $1 \leq i \leq j-2$, the following inequality holds:

$$
\sigma_{M}\left(G_{i}, G_{j}\right) \geq \sigma_{M}\left(G_{i+1}, G_{j}\right)
$$

wherefrom one obtains

$$
\sigma_{M}\left(G_{1}, G_{j}\right) \geq \sigma_{M}\left(G_{2}, G_{j}\right) \geq \sigma_{M}\left(G_{3}, G_{j}\right) \geq \cdots \geq \sigma_{M}\left(G_{j-1}, G_{j}\right),
$$

and the cospectralities can be calculated.

Theorem 2.5. Let $\mathcal{T}^{\circ}=\left\{K_{1, n}\right\} \cup \mathcal{T}$ be the set of trees of order $n+1$, where $n \geq 3$ and $\mathcal{T}$ is the set of trees with $n-1$ pendant vertices. The parameters of the A-spectral distances of graphs from the set $\mathcal{T}^{\circ}$ are given by Proposition 2.4.

Proof. Let $G_{1}, G_{2}, \ldots, G_{\left\lceil\frac{n}{2}\right\rceil}$ be the sequence of graphs given by $G_{1}=K_{1, n}$ and $G_{i}=K_{1, n-i+1} \cdot K_{1, i-1}$, for $i=2,3, \ldots,\left\lceil\frac{n}{2}\right\rceil$. This means that $\mathcal{T}=\left\{G_{2}, \ldots, G_{\left\lceil\frac{n}{2}\right\rceil}\right\}$. Using Theorem 1.3 one finds the characteristic polynomial of $G_{i}$ with respect to the adjacency matrix:

$$
P_{G_{i}}(x)=x^{n-3}\left(x^{4}-n x^{2}+(n-i)(i-1)\right) .
$$

Then, the spectrum of $G_{i}$ is $\pm \lambda_{1}\left(G_{i}\right), \pm \lambda_{2}\left(G_{i}\right)$, and $[0]^{n-3}$, where $\lambda_{1}\left(G_{i}\right)=$ $\sqrt{\frac{n}{2}+\frac{1}{2} \sqrt{n^{2}-4(n-i)(i-1)}}$ and $\lambda_{2}\left(G_{i}\right)=\sqrt{\frac{n}{2}-\frac{1}{2} \sqrt{n^{2}-4(n-i)(i-1)}}$. It can 
be checked that $\lambda_{1}\left(G_{i}\right) \geq \lambda_{1}\left(G_{j}\right)$ and $\lambda_{2}\left(G_{i}\right) \leq \lambda_{2}\left(G_{j}\right)$, for $1 \leq i<j \leq\left\lceil\frac{n}{2}\right\rceil$, so the $A$-spectral distance between $G_{i}$ and $G_{j}, 1 \leq i<j \leq\left\lceil\frac{n}{2}\right\rceil$, is $\sigma_{A}\left(G_{i}, G_{j}\right)=\sum_{k=1}^{n+1}\left|\lambda_{k}\left(G_{i}\right)-\lambda_{k}\left(G_{j}\right)\right|=2\left(\lambda_{1}\left(G_{i}\right)-\lambda_{1}\left(G_{j}\right)\right)+2\left(\lambda_{2}\left(G_{j}\right)-\lambda_{2}\left(G_{i}\right)\right)$.

Remark 2.6. It is obvious that the parameters of the $A$-spectral distances of graphs from the set $\mathcal{T}$ are also given by Proposition 2.4 .

The graphs from the set $\mathcal{T}^{\circ}$ for $n=7$ are presented in Figure 1 . Their adjacency eigenvalues (rounded to two decimal places) are: $\operatorname{Spect}\left(G_{1}\right): 2.65,[0]^{6},-2.65$; $\operatorname{Spect}\left(G_{2}\right): 2.49,0.90,[0]^{4},-0.90,-2.49 ; \operatorname{Spect}\left(G_{3}\right): 2.36,1.20,[0]^{4},-1.20,-2.36$, and $\operatorname{Spect}\left(G_{4}\right): 2.30,1.30,[0]^{4},-1.30,-2.30$. So, the $A$-spectral distances between these graphs are approximately equal to: $\sigma_{A}\left(G_{1}, G_{2}\right)=2.12, \sigma_{A}\left(G_{1}, G_{3}\right)=2.98$, $\sigma_{A}\left(G_{1}, G_{4}\right)=3.30, \sigma_{A}\left(G_{2}, G_{3}\right)=0.86, \sigma_{A}\left(G_{2}, G_{4}\right)=1.18$ and $\sigma_{A}\left(G_{3}, G_{4}\right)=0.32$.

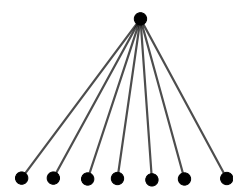

$G_{1}$

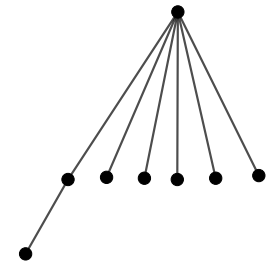

$G_{2}$

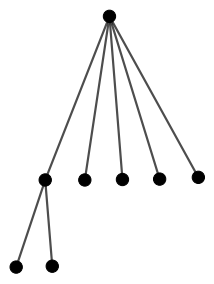

$G_{3}$

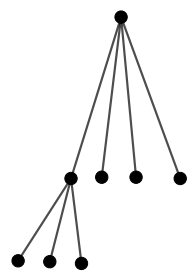

$G_{4}$

Figure 1. Trees from the set $\mathcal{T}^{\circ}$

Let $\mathcal{T}^{*}=\left\{G_{0}, G_{1}, \ldots, G_{\frac{n}{2}}\right\}$ be the set of graphs of order $n+1$, where $n>4$ is even, $G_{0}=K_{1, n}$ and $G_{i}=\left(\left((K_{1, n-i} \cdot \underbrace{\left.\left.\left.K_{2}\right) \cdot K_{2}\right) \cdots\right) \cdot K_{2}}_{i}\right.\right.$, for $1 \leq i \leq \frac{n}{2}$. Here, for fixed $i$, by the graph operation of coalescence, a vertex of the $j$-th graph $K_{2}$ is identified with a pendant vertex of the graph $\left(\left((K_{1, n-i} \cdot \underbrace{\left.\left.\left.K_{2}\right) \cdot K_{2}\right) \cdots\right) \cdot K_{2}}_{j-1}\right.\right.$, which is adjacent to the vertex of the maximum degree (i.e. $n-i$ ) in this graph, for each $1 \leq j \leq i$. It can be noticed that the graphs from the set $\mathcal{T}^{*}$ are such trees that $G_{i}$ has $i$ vertices of degree 2 , one vertex of degree $n-i$ and $n-i$ pendant vertices. The graphs from $\mathcal{T}^{*}$ for $n=8$ are presented in Figure 2

In order to determine the characteristic polynomials of the graphs from the set $\mathcal{T}^{*}$ regarding the adjacency matrix, one can use $G_{i}^{(m)}$ to denote the graph $G_{i}$ whose order is $m$, i.e. $G_{i}^{(m)}=\left(\left((K_{1, m-1-i} \cdot \underbrace{\left.\left.\left.K_{2}\right) \cdot K_{2}\right) \cdots\right) \cdot K_{2}}_{i}\right.\right.$, where $m$ is an odd positive 


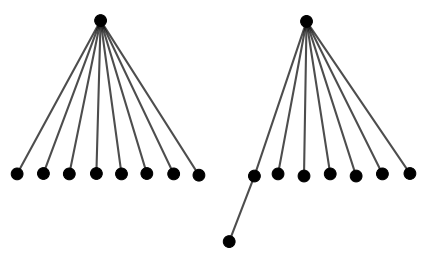

$G_{0}$

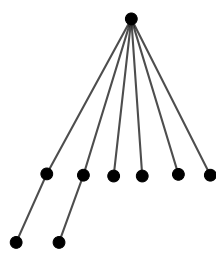

$G_{2}$

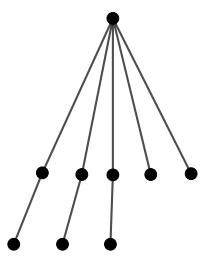

$G_{3}$

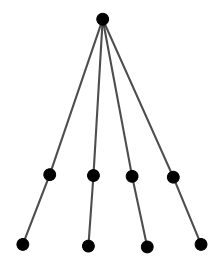

$G_{4}$

Figure 2. Trees from the set $\mathcal{T}^{*}$

integer, and $G_{i}^{(m-j)}, 1 \leq j \leq m-1-2 i$, to denote the graph obtained from $G_{i}^{(m)}$ by deleting $j$ vertices of degree 1 . Therefore, both $G_{i}^{(m)}$ and $G_{i}^{(m-j)}$ have $i$ vertices whose degree is equal to 2.

Lemma 2.7. The characteristic polynomials of the graphs from the set $\mathcal{T}^{*}$, with respect to the adjacency matrix, are given by the recurrent formula

$$
\begin{aligned}
& P_{G_{0}^{(n+1)}}(x)=P_{K_{1, n}}(x)=\left(x^{2}-n\right) x^{n-1} \\
& P_{G_{i}^{(n+1)}}(x)=x P_{G_{i-1}^{(n)}}(x)-P_{G_{i-1}^{(n-1)}}(x), \quad 1 \leq i \leq \frac{n}{2} .
\end{aligned}
$$

Proof. Since $G_{i}^{(n+1)}=\left(\left((K_{1, n-i} \cdot \underbrace{\left.\left.\left.K_{2}\right) \cdot K_{2}\right) \cdots\right) \cdot K_{2}}_{i}\right.\right.$, i.e. $G_{i}^{(n+1)}=G_{i-1}^{(n)} \cdot K_{2}$, where a vertex of $K_{2}$ is identified with a pendant vertex of $G_{i-1}^{(n)}$ which is adjacent to the vertex of the maximum degree in this graph, according to Theorem 1.3 , one can get:

$$
\begin{aligned}
P_{G_{i}^{(n+1)}}(x) & =P_{G_{i-1}^{(n)}}(x) P_{K_{1}}(x)+P_{G_{i-1}^{(n-1)}}(x) P_{K_{2}}(x)-x P_{G_{i-1}^{(n-1)}}(x) P_{K_{1}}(x) \\
& =x P_{G_{i-1}^{(n)}}(x)-P_{G_{i-1}^{(n-1)}}(x) .
\end{aligned}
$$

The explicit formula for the characteristic polynomial of $G_{i}^{(n+1)}$ is presented below.

Lemma 2.8. The characteristic polynomial of the graph $G_{i}^{(n+1)}, 1 \leq i \leq \frac{n}{2}$, from $\mathcal{T}^{*}$ with respect to the adjacency matrix is

$$
P_{G_{i}^{(n+1)}}(x)=\sum_{k=0}^{i}(-1)^{k}\left(\begin{array}{l}
i \\
k
\end{array}\right) x^{i-k} P_{G_{0}^{(n-i-k+1)}}(x) .
$$

Proof. The following equality holds:

$$
(-1)^{0}\left(\begin{array}{l}
0 \\
0
\end{array}\right) x^{0-0} P_{G_{0}^{(n+1)}}(x)=P_{G_{0}^{(n+1)}}(x),
$$


where it is supposed that $\left(\begin{array}{l}0 \\ 0\end{array}\right)=1$. Further, one finds:

$$
\begin{aligned}
& x P_{G_{i-1}^{(n)}}(x)-P_{G_{i-1}^{(n-1)}}(x) \\
& =\sum_{k=0}^{i-1}(-1)^{k}\left(\begin{array}{c}
i-1 \\
k
\end{array}\right) x^{i-k} P_{G_{0}^{(n-i+1-k)}}(x) \\
& \quad+\sum_{k=0}^{i-1}(-1)^{k+1}\left(\begin{array}{c}
i-1 \\
k
\end{array}\right) x^{i-1-k} P_{G_{0}^{(n-i-k)}}(x) \\
& =x^{i} P_{G_{0}^{(n-i+1)}}(x)+\sum_{k=1}^{i-1}(-1)^{k}\left(\begin{array}{c}
i-1 \\
k
\end{array}\right) x^{i-k} P_{G_{0}^{(n-i+1-k)}}(x) \\
& \quad+(-1)^{i} P_{G_{0}^{(n-2 i+1)}}(x)+\sum_{k=1}^{i-1}(-1)^{k}\left(\begin{array}{c}
i-1 \\
k-1
\end{array}\right) x^{i-k} P_{G_{0}^{(n-i+1-k)}}(x) \\
& =x^{i} P_{G_{0}^{(n-i+1)}}(x)+(-1)^{i} P_{G_{0}^{(n-2 i+1)}}(x)+\sum_{k=1}^{i-1}(-1)^{k}\left(\begin{array}{c}
i \\
k
\end{array}\right) x^{i-k} P_{G_{0}^{(n-i+1-k)}}(x) \\
& =\sum_{k=0}^{i}(-1)^{k}\left(\begin{array}{c}
i \\
k
\end{array}\right) x^{i-k} P_{G_{0}^{(n-i+1-k)}}(x)=P_{G_{i}^{(n+1)}}(x) .
\end{aligned}
$$

Lemma 2.9. The adjacency eigenvalues of the graph $G_{i}^{(n+1)}, 1 \leq i<\frac{n}{2}$, from the set of graphs $\mathcal{T}^{*}$ are: $[1]^{i-1},[0]^{n-(2 i+1)},[-1]^{i-1}$, and four eigenvalues which are the roots of the polynomial $Q_{i}^{(n+1)}(x)=x^{4}+(-n-1+i) x^{2}+(n-2 i)$.

Remark 2.10. The adjacency spectrum of the graph $G_{\frac{n}{2}}^{(n+1)}$ is $\pm \sqrt{\frac{n}{2}+1},[1]^{i-1}$, $0,[-1]^{i-1}$.

Proof. The characteristic polynomial of $G_{1}^{(n+1)}$ regarding the adjacency matrix is

$$
\begin{aligned}
P_{G_{1}^{(n+1)}}(x) & =x^{n-3} \sum_{k=0}^{1}(-1)^{k}\left(\begin{array}{l}
1 \\
k
\end{array}\right) x^{2(1-k)}\left(x^{2}-n+1+k\right) \\
& =x^{n-3}\left(x^{4}-n x^{2}+n-2\right) \\
& =x^{n-3} Q_{1}^{(n+1)}(x)
\end{aligned}
$$

so the spectrum of $G_{1}^{(n+1)}$ consists of $n-3$ eigenvalues which are equal to zero, while the remaining four eigenvalues, different from \pm 1 , are the roots of the polynomial $Q_{1}^{(n+1)}(x)$. 
The characteristic polynomial of $G_{2}^{(n+1)}$ with respect to the adjacency matrix is

$$
\begin{aligned}
P_{G_{2}^{(n+1)}}(x) & =x^{n-5} \sum_{k=0}^{2}(-1)^{k}\left(\begin{array}{l}
2 \\
k
\end{array}\right) x^{2(2-k)}\left(x^{2}-n+2+k\right) \\
& =x^{n-5}\left(x^{6}-n x^{4}+(2 n-5) x^{2}-n+4\right) \\
& =x^{n-5}\left(x^{2}-1\right)\left(x^{4}+(-n+1) x^{2}+(n-4)\right) \\
& =x^{n-5}\left(x^{2}-1\right) Q_{2}^{(n+1)}(x)
\end{aligned}
$$

Therefore, the spectrum of $G_{2}^{(n+1)}$ is $[0]^{n-5}, 1,-1$, while the remaining four eigenvalues are the roots of the polynomial $Q_{2}^{(n+1)}(x)$.

It can be assumed that in the adjacency spectrum of the graph $G_{l}^{(n+1)}$ there are $n-2 l-1$ eigenvalues which are equal to zero, $l-1$ eigenvalues which are equal to 1 , $l-1$ eigenvalues which are equal to -1 , and that the remaining four eigenvalues are the roots of the polynomial

$$
Q_{l}^{(n+1)}(x)=x^{4}+(-n-1+l) x^{2}+(n-2 l)
$$

i.e. that the characteristic polynomial of the graph $G_{l}^{(n+1)}$ is

$$
P_{G_{l}^{(n+1)}}(x)=x^{n-(2 l+1)}\left(x^{2}-1\right)^{l-1} Q_{l}^{(n+1)}(x) .
$$

The characteristic polynomial of the graph $G_{l+1}^{(n+1)}$, according to 2.2 , satisfies the relation

$$
P_{G_{l+1}^{(n+1)}}(x)=x P_{G_{l}^{(n)}}(x)-P_{G_{l}^{(n-1)}}(x),
$$

wherefrom, by using (2.4), one can get

$$
P_{G_{l+1}^{(n+1)}}(x)=x^{n-2 l-3}\left(x^{2}-1\right)^{l-1}\left(x^{2} Q_{l}^{(n)}(x)-Q_{l}^{(n-1)}(x)\right) .
$$

Considering 2.3 , the previous relation becomes:

$$
\begin{aligned}
& P_{G_{l+1}^{(n+1)}}(x) \\
& \quad=x^{n-2 l-3}\left(x^{2}-1\right)^{l-1}\left(x^{6}+(-n+l-1) x^{4}+(2 n-2-3 l) x^{2}-n+2+2 l\right) \\
& \quad=x^{n-2 l-3}\left(x^{2}-1\right)^{l}\left(x^{4}+(l-n) x^{2}+n-2 l-2\right) \\
& \quad=x^{n-2 l-3}\left(x^{2}-1\right)^{l} Q_{l+1}^{(n+1)}(x),
\end{aligned}
$$

which means that the statement is proved by using the principle of mathematical induction. 
The roots of the polynomial $Q_{i}^{(n+1)}(x)$ are

$$
\begin{aligned}
& x_{1}=\sqrt{\frac{(n+1-i)+\sqrt{(-n-1+i)^{2}-4(n-2 i)}}{2}}, \\
& x_{2}=\sqrt{\frac{(n+1-i)-\sqrt{(-n-1+i)^{2}-4(n-2 i)}}{2}}, \\
& x_{3}=-x_{1}, \\
& x_{4}=-x_{2} .
\end{aligned}
$$

By direct computation, it can be checked that $x_{1}>1$ and $x_{2}<1$, i.e. $x_{3}<-1$ and $x_{4}>-1$.

In [13, the authors consider the graphs $G_{k, l}(1 \leq k \leq l)$ and $G_{k-1, l+1}$, defined as follows: let $G$ be a connected graph with at least two vertices and let $v$ be an arbitrary vertex. $G_{k, l}$ is the graph obtained by coalescing $G$ with two new paths, $P_{k+1}$ and $Q_{l+1}$, precisely $P_{k+1}: v\left(\triangleq v_{0}\right) v_{1} v_{2} \cdots v_{k}$ and $Q_{l+1}: v\left(\triangleq v_{0}\right) u_{1} u_{2} \cdots u_{l}$, by identifying an end vertex of $P_{k+1}$ and an end vertex of $Q_{l+1}$, both with the same vertex $v\left(\triangleq v_{0}\right)$ of $G$. Then, $G_{k-1, l+1}=G_{k, l}-v_{k-1} v_{k}+u_{l} v_{k}$. In this regard, the authors state the following lemma from [12].

Lemma 2.11 (13, Lemma 2.1]). Let $G$ be a connected graph on $n(n \geq 2)$ vertices and $v\left(\triangleq v_{0}\right)$ be a vertex of $G$, and let $G_{k, l}(l \geq k \geq 1)$ be the graph defined as above. Then,

$$
\lambda_{1}\left(G_{k, l}\right)>\lambda_{1}\left(G_{k-1, l+1}\right) .
$$

It can be noticed that for each $0 \leq i \leq \frac{n}{2}-1$, the graph $G_{i}^{(n+1)}$ from the set $\mathcal{T}^{*}$ is, in fact, the graph $G_{k, l}$ for $k=l=1$, where $v\left(\triangleq v_{0}\right)$ is the vertex whose degree is $n-i$ in $G_{i}$. Therefore, $G_{i+1}^{(n+1)}\left(=G_{k-1, l+1}\right)$ is obtained from $G_{i}^{(n+1)}$ in the manner described above. So, according to Lemma 2.11, it follows that $\lambda_{1}\left(G_{i}^{(n+1)}\right)>\lambda_{1}\left(G_{j}^{(n+1)}\right)$, for $1 \leq i<j \leq \frac{n}{2}$.

Now, the $A$-spectral distances of the graphs from $\mathcal{T}^{*}$ are

$$
\begin{aligned}
& \sigma_{A}\left(G_{0}, G_{i}\right)=2\left(\sqrt{n+1}-\lambda_{1}\left(G_{i}\right)+(i-1)+\lambda_{2}\left(G_{i}\right)\right), \quad 1 \leq i<\frac{n}{2} ; \\
& \sigma_{A}\left(G_{0}, G_{\frac{n}{2}}\right)=2\left(\sqrt{n+1}-\lambda_{1}\left(G_{\frac{n}{2}}\right)\right)+n-2 ; \\
& \sigma_{A}\left(G_{i}, G_{j}\right)=2\left(\lambda_{1}\left(G_{i}\right)-\lambda_{2}\left(G_{i}\right)-\lambda_{1}\left(G_{j}\right)+\lambda_{2}\left(G_{j}\right)+j-i\right), \quad 1 \leq i<j<\frac{n}{2} ; \\
& \sigma_{A}\left(G_{i}, G_{\frac{n}{2}}\right)=2\left(\lambda_{1}\left(G_{i}\right)-\lambda_{2}\left(G_{i}\right)-\lambda_{1}\left(G_{\frac{n}{2}}\right)+\frac{n}{2}-i\right), \quad 1 \leq i<\frac{n}{2} .
\end{aligned}
$$

By direct computation, one can verify that the $A$-spectral distances between graphs from the set $\mathcal{T}^{*}$ satisfy (2.1). In that way, the following theorem has been proved.

Theorem 2.12. The parameters of the A-spectral distances of graphs from the set $\mathcal{T}^{*}$ are given by Proposition 2.4

The adjacency eigenvalues of graphs presented in Figure 2 (rounded to two decimal places) are: $\operatorname{Spect}\left(G_{0}\right): 2.83,[0]^{7},-2.83 ; \operatorname{Spect}\left(G_{1}\right): 2.68,0.91,[0]^{5}$, 
$-0.91,-2.68 ; \operatorname{Spect}\left(G_{2}\right): 2.52,1,0.79,[0]^{3},-0.79,-1,-2.52 ; \operatorname{Spect}\left(G_{3}\right): 2.38$, $[1]^{2}, 0.59,0,-0.59,[-1]^{2},-2.38$, and $\operatorname{Spect}\left(G_{4}\right): 2.24,[1]^{3}, 0,[-1]^{3},-2.24$. Therefore, the $A$-spectral distances between these graphs are approximately equal to: $\sigma_{A}\left(G_{0}, G_{1}\right)=2.12, \sigma_{A}\left(G_{0}, G_{2}\right)=4.2, \sigma_{A}\left(G_{0}, G_{3}\right)=6.08, \sigma_{A}\left(G_{0}, G_{4}\right)=7.18$, $\sigma_{A}\left(G_{1}, G_{2}\right)=2.08, \sigma_{A}\left(G_{1}, G_{3}\right)=3.96, \sigma_{A}\left(G_{1}, G_{4}\right)=5.06, \sigma_{A}\left(G_{2}, G_{3}\right)=1.88$, $\sigma_{A}\left(G_{2}, G_{4}\right)=2.98$, and $\sigma_{A}\left(G_{3}, G_{4}\right)=1.1$.

The following lemma and conjecture appear in [9].

Lemma 2.13 ([9, Lemma 5.1]). If $T_{n}$ is an arbitrary tree of order $n$, then

$$
\sigma_{A}\left(T_{n}, K_{1, n-1}\right) \leq \sigma_{A}\left(P_{n}, K_{1, n-1}\right) .
$$

Conjecture 2.14 ([9. Conjecture 4]). The A-spectral distance between any two trees of order $n$ does not exceed $\sigma_{A}\left(P_{n}, K_{1, n-1}\right)$.

The next statement supports Conjecture 2.14

Proposition 2.15. Let $T_{n}$ and $T_{n}^{*}$ be two trees of order $n$ such that $\lambda_{i}\left(T_{n}\right)<$ $\lambda_{i}\left(T_{n}^{*}\right) \leq 2 \lambda_{i}\left(T_{n}\right)$, for $i=2,3, \ldots,\left\lfloor\frac{n}{2}\right\rfloor$. Then $\sigma_{A}\left(T_{n}, T_{n}^{*}\right) \leq \sigma_{A}\left(P_{n}, K_{1, n-1}\right)$.

Proof. The following equality holds:

$$
\sigma_{A}\left(T_{n}, K_{1, n-1}\right)=2\left(\lambda_{1}\left(K_{1, n-1}\right)-\lambda_{1}\left(T_{n}\right)\right)-2 \lambda_{1}\left(T_{n}\right)+E\left(T_{n}\right),
$$

so one can obtain:

$$
\begin{aligned}
\sigma_{A}\left(T_{n}, T_{n}^{*}\right) & =2\left|\lambda_{1}\left(T_{n}\right)-\lambda_{1}\left(T_{n}^{*}\right)\right|+2 \sum_{i=2}^{\left\lfloor\frac{n}{2}\right\rfloor}\left|\lambda_{i}\left(T_{n}\right)-\lambda_{i}\left(T_{n}^{*}\right)\right| \\
& \leq 2\left|\lambda_{1}\left(K_{1, n-1}\right)-\lambda_{1}\left(T_{n}\right)\right|+2 \sum_{i=2}^{\left\lfloor\frac{n}{2}\right\rfloor}\left|\lambda_{i}\left(T_{n}\right)\right| \\
& =2\left(\lambda_{1}\left(K_{1, n-1}\right)-\lambda_{1}\left(T_{n}\right)\right)+E\left(T_{n}\right)-2 \lambda_{1}\left(T_{n}\right)=\sigma_{A}\left(T_{n}, K_{1, n-1}\right),
\end{aligned}
$$

since $\lambda_{1}\left(K_{1, n-1}\right) \geq \lambda_{1}\left(T_{n}\right)$ for every tree $T_{n}$ of order $n$. The previous inequality together with Lemma 2.13 means that

$$
\sigma_{A}\left(T_{n}, T_{n}^{*}\right) \leq \sigma_{A}\left(T_{n}, K_{1, n-1}\right) \leq \sigma_{A}\left(P_{n}, K_{1, n-1}\right) .
$$

3. Examples of gRAPHS Whose SPECTRAL Distances With RESPECT TO THE ADJACENCY, THE LAPLACIAN AND THE SIGNLESS LAPLACIAN MATRIX ARE

$$
\text { MUTUALLY EQUAL }
$$

In [10], an example of a graph whose $A$-, $L$ - and $Q$-spectral distances are mutually equal is presented. Precisely, the following proposition has been proved.

Proposition 3.1 ([10, Proposition 2.2]). Let $O(G)$ stand for the application of line and complement of a regular graph $G$ an arbitrary number of times and in an arbitrary order. Let $G_{1}$ and $G_{2}$ be r-regular graphs on $n$ vertices. Then

$$
\begin{aligned}
\sigma_{A}\left(O\left(G_{1}\right), O\left(G_{2}\right)\right) & =\sigma_{A}\left(G_{1}, G_{2}\right)=\sigma_{L}\left(O\left(G_{1}\right), O\left(G_{2}\right)\right) \\
& =\sigma_{L}\left(G_{1}, G_{2}\right)=\sigma_{Q}\left(O\left(G_{1}\right), O\left(G_{2}\right)\right)=\sigma_{Q}\left(G_{1}, G_{2}\right) .
\end{aligned}
$$


In this section, some new families of graphs whose $A$-spectral distances are equal to the corresponding $L$ - and $Q$-spectral distances will be considered. First, let us recall that the graph $G_{2}$ is an edges-deleted subgraph of the graph $G_{1}$ if it is obtained by deleting some edges of $G_{1}$ and that the following result holds.

Proposition 3.2 ([10, Proposition 3.1]). Let $G_{1}$ be a graph on $n$ vertices. If $G_{2}$ is obtained by deleting $k$ edges from $G_{1}$, then $\sigma_{L}\left(G_{1}, G_{2}\right)=\sigma_{Q}\left(G_{1}, G_{2}\right)=2 k$.

Proposition 3.3. Let $G_{1}$ and $G_{2}$ be two graphs of order $n$ such that $G_{2}$ is an edges-deleted subgraph of $G_{1}$, and $\varepsilon\left(G_{1}\right)-\varepsilon\left(G_{2}\right)=k$, where $\varepsilon\left(G_{i}\right), i=1,2$, is the number of edges of the graph $G_{i}$. Let the $A$-spectra of these graphs be $\lambda_{1}\left(G_{i}\right) \geq$ $\lambda_{2}\left(G_{i}\right) \geq \cdots \geq \lambda_{n}\left(G_{i}\right), i=1,2$, and let $I \subseteq\{1,2, \ldots, n\}$ be the set of indices $i$ such that $\lambda_{i}\left(G_{2}\right) \geq \lambda_{i}\left(G_{1}\right)$. If $\sum_{i \in I}\left(\lambda_{i}\left(G_{2}\right)-\lambda_{i}\left(G_{1}\right)\right)=k$, then

$$
\sigma_{A}\left(G_{1}, G_{2}\right)=\sigma_{L}\left(G_{1}, G_{2}\right)=\sigma_{Q}\left(G_{1}, G_{2}\right)=2 k .
$$

Proof. The spectral distance between $G_{1}$ and $G_{2}$ regarding the adjacency matrix is equal to

$$
\begin{aligned}
\sigma_{A}\left(G_{1}, G_{2}\right) & =\sum_{i \in I}\left(\lambda_{i}\left(G_{2}\right)-\lambda_{i}\left(G_{1}\right)\right)+\sum_{i \in\{1,2, \ldots, n\} \backslash I}\left(\lambda_{i}\left(G_{1}\right)-\lambda_{i}\left(G_{2}\right)\right) \\
& =2 \sum_{i \in I}\left(\lambda_{i}\left(G_{2}\right)-\lambda_{i}\left(G_{1}\right)\right)+\sum_{i=1}^{n} \lambda_{i}\left(G_{1}\right)-\sum_{i=1}^{n} \lambda_{i}\left(G_{2}\right) \\
& =2 k,
\end{aligned}
$$

since the sum of the adjacency eigenvalues of a graph is equal to zero. The remaining two equalities follow from Proposition 3.2 .

Let $\mathcal{K}=\left\{G_{0}, G_{1}, \ldots, G_{q}\right\}$ be the set of graphs of order $n+1$ such that $G_{0}=K_{1, n}$ and $G_{i}=K_{1,(n-i m)} \cdot H_{i}, i=1,2, \ldots, q$. Here, $H_{i}=K_{1} \nabla i K_{m}$; by the graph operation ', the vertex of maximum degree in the graph $K_{1,(n-i m)}$ is identified with the vertex of maximum degree in the graph $H_{i} ; n \equiv 0(\bmod m)$, i.e. $n=m q$ for $q \in \mathbb{Z}^{+} ; n>m$ and $m>1$. Graph $G_{2}$ from $\mathcal{K}$ for $n=16$ and $m=4$ is presented in Figure 3 .

To prove Proposition 3.6 which is related to the adjacency spectral distances of graphs from the set $\mathcal{K}$, one needs the following results.

Corollary 3.4 (Interlacing Theorem, [5, Corollary 1.3.12]). Let $G$ be a graph with $n$ vertices and eigenvalues $\lambda_{1} \geq \lambda_{2} \geq \cdots \geq \lambda_{n}$, and let $H$ be an induced subgraph of $G$ with $m$ vertices. If the eigenvalues of $H$ are $\mu_{1} \geq \mu_{2} \geq \cdots \geq \mu_{m}$, then $\lambda_{n-m+i} \leq \mu_{i} \leq \lambda_{i}, i=1,2, \ldots, m$.

Proposition 3.5 ([5, Proposition 1.3.10]). If $G-u v$ is the graph obtained from a connected graph $G$ by deleting the edge uv, then $\lambda_{1}(G-u v)<\lambda_{1}(G)$. 


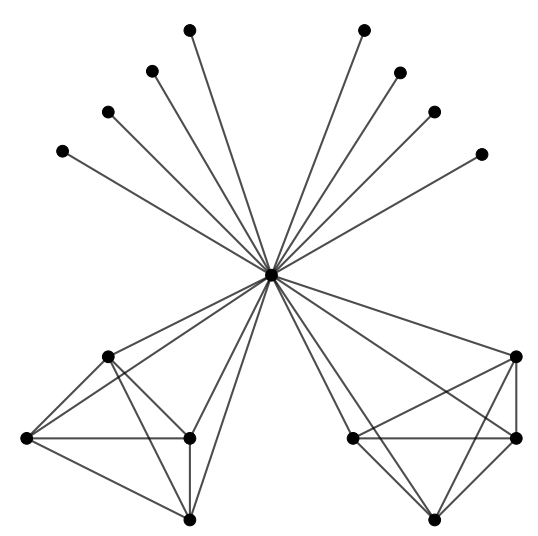

Figure 3. Graph $G_{2}$ from $\mathcal{K}$ for $n=16$ and $m=4$

Proposition 3.6. The following holds:

(1) Let $G_{i}, G_{j}$ and $G_{k}$ be three arbitrary graphs from the set $\mathcal{K}$, such that $1 \leq i<j<k \leq q$. Then the A-spectral distances of these graphs satisfy (2.1).

(2) The $A$-, $L$ - and $Q$-spectral distances of graphs from $\mathcal{K}$ are mutually equal for $m=2$.

Proof. By applying Theorem 1.2 , the characteristic polynomial of the graph $H_{i}=$ $K_{1} \nabla i K_{m}$, with respect to the adjacency matrix, can be obtained:

$$
P_{H_{i}}(x)=(x-m+1)^{i-1}(x+1)^{i(m-1)}\left(x^{2}-(m-1) x-i m\right) .
$$

According to Theorem 1.3 , the characteristic polynomial of the graph $G_{i}$, regarding the adjacency matrix, is

$$
P_{G_{i}}(x)=x^{n-i m-1}(x-(m-1))^{i-1}(x+1)^{i(m-1)} Q_{i}(x),
$$

where $Q_{i}(x)=x^{3}-(m-1) x^{2}-n x+(m-1)(n-i m)$. Let $x_{1}, x_{2}$ and $x_{3}$ be the roots of the polynomial $Q_{i}(x)$.

According to Corollary 3.4, as $K_{m}$ is an induced subgraph of $G_{i}$, for each $1 \leq$ $i \leq q, \lambda_{1}\left(K_{m}\right)=m-1 \leq \lambda_{1}\left(G_{i}\right)$. Since $Q_{i}(m-1)=-i m(m-1) \neq 0$, it follows that $\lambda_{1}\left(G_{i}\right)>m-1$, so $x_{1}=\lambda_{1}\left(G_{i}\right)$, for $1 \leq i \leq q$.

Graph $H_{i}$ is an induced subgraph of $G_{i}$, for each $1 \leq i<q$, and from (3.1) it follows that its $A$-spectrum is $h_{1}=\frac{1}{2}\left(m-1+\sqrt{(m-1)^{2}+4 i m}\right)>m-1$, $[m-1]^{i-1},[-1]^{i(m-1)}, h_{2}=\frac{1}{2}\left(m-1-\sqrt{(m-1)^{2}+4 i m}\right) \leq-1$. Applying Corollary 3.4 results in:

$$
\lambda_{n+1}\left(G_{i}\right) \leq \lambda_{i m+1}\left(H_{i}\right)=h_{2} \leq-1, \quad \text { for } 1 \leq i<q .
$$


For $i=q, K_{1} \nabla q K_{m-1}$ is an induced subgraph of $G_{q}$. Since

$P_{K_{1} \nabla q K_{m-1}}(x)=(x-m+2)^{q-1}(x+1)^{q(m-2)}\left(x^{2}-(m-2) x-q(m-1)\right)$,

the $A$-spectrum of $K_{1} \nabla q K_{m-1}$ consists of the following eigenvalues: $[m-2]^{q-1}$, $[-1]^{q(m-2)}, g_{1}=\frac{1}{2}\left(m-2+\sqrt{(m-2)^{2}+4 q(m-1)}\right)$, where $g_{1}>m-2$, and $g_{2}=\frac{1}{2}\left(m-2-\sqrt{(m-2)^{2}+4 q(m-1)}\right)$, where $g_{2}<-1$. So, reapplying Corollary 3.4 results in

$$
\lambda_{n+1}\left(G_{q}\right) \leq \lambda_{1+q(m-1)}\left(K_{1} \nabla q K_{m-1}\right)<-1 .
$$

From (3.2) and (3.3), $x_{3}=\lambda_{n+1}\left(G_{i}\right) \leq-1$ is finally found.

Since $Q_{i}(0)=(m-1)(n-i m)>0$ and $Q_{i}(m-1)=-i m(m-1)<0$, for each $i \neq q$, the eigenvalue $x_{2}$ of the graph $G_{i}$ is in the interval $(0, m-1)$.

If $i=q$, then $Q_{q}(x)=x\left(x^{2}-(m-1) x-n\right)$, which means that the adjacency spectrum of $G_{q}$ is $\frac{1}{2}\left(m-1+\sqrt{(m-1)^{2}+4 n}\right),[m-1]^{q-1},[-1]^{n-q}$, $\frac{1}{2}\left(m-1-\sqrt{(m-1)^{2}+4 n}\right)$.

According to Proposition $3.5 x_{1}\left(G_{i}\right)<x_{1}\left(G_{j}\right)$, i.e. $\lambda_{1}\left(G_{i}\right)<\lambda_{1}\left(G_{j}\right)$ is found, where $1 \leq i<j \leq q$. The functions $Q_{i}(x)$ and $Q_{j}(x)$ differ only in the positive coefficient with $x^{0}$ and they have no common points, which means that one of them is the expansion (shrinking) of the other in a certain interval. The function $Q_{i}(x), 1 \leq i \leq q$, is positive in the interval $\left(x_{2}, x_{3}\right)$, it is monotonically increasing on $\left(-\infty, \frac{1}{3}\left(m-1-\sqrt{(m-1)^{2}+3 n}\right)\right)$, and it attains the maximum for $x=$ $\frac{1}{3}\left(m-1-\sqrt{(m-1)^{2}+3 n}\right)$. Hence,

$$
Q_{i}\left(\frac{1}{3}\left(m-1-\sqrt{(m-1)^{2}+3 n}\right)\right)>Q_{j}\left(\frac{1}{3}\left(m-1-\sqrt{(m-1)^{2}+3 n}\right)\right),
$$

and also $x_{3}<\frac{1}{3}\left(m-1-\sqrt{(m-1)^{2}+3 n}\right)$. Therefore, $x_{3}\left(G_{j}\right)>x_{3}\left(G_{i}\right)$, i.e. $\lambda_{n+1}\left(G_{j}\right)>\lambda_{n+1}\left(G_{i}\right)$, for $1 \leq i<j \leq q$.

For $G_{i}, G_{j} \in \mathcal{K}$, where $1 \leq i<j \leq q$, the following holds:

$$
\sum_{k \in\{n-(m-1) j+1, \ldots, n-(m-1) i+1\}}\left(\lambda_{k}\left(G_{i}\right)-\lambda_{k}\left(G_{j}\right)\right)=(m-1)(j-i)
$$

and

$$
\varepsilon\left(G_{j}\right)-\varepsilon\left(G_{i}\right)=\frac{m(m-1)}{2}(j-i) .
$$

The relations (3.4) and 3.5 and Proposition 3.3 imply that the $A-, L$ - and $Q$-spectral distances of graphs from the set $\mathcal{K}$ are mutually equal for $m=2$ (see Figure 4).

Otherwise, the $A$-spectral distance of graphs $G_{i}$ and $G_{j}$, for $1 \leq i<j \leq q$, is equal to

$$
\sigma_{A}\left(G_{i}, G_{j}\right)=2(m-1)(j-i),
$$

wherefrom it can be checked that (2.1) is satisfied. 

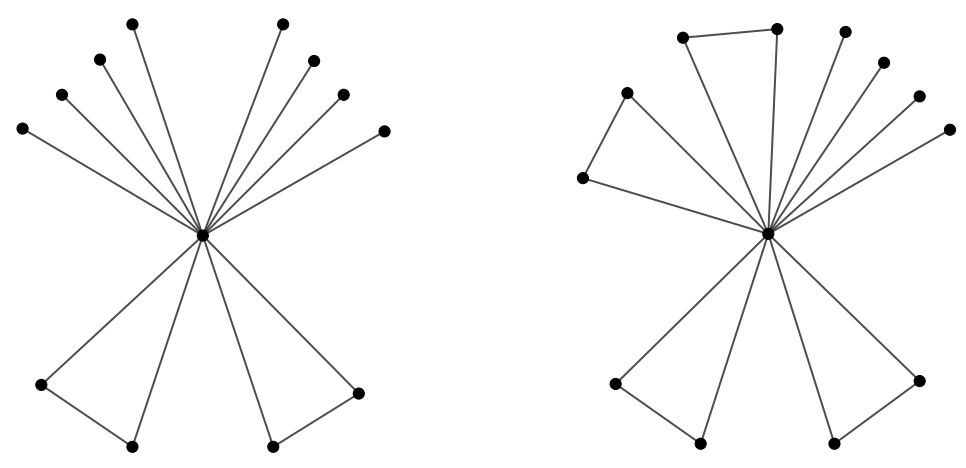

Figure 4. Example of graphs whose $A$-, $L$ - and $Q$ - spectral distances are equal

There are examples of graphs such that the $A$-spectral distance between them and their edge-deleted subgraphs is equal to a small constant. For example, in [9] it was proved that $\sigma_{A}\left(C_{n}, P_{n}\right)=\sigma_{A}\left(C_{n}, C_{n}-e\right)=2$, while in [1] it was proved that $\sigma_{A}\left(K_{n}, K_{n}-e\right)=2$, for $n \geq 2$. Here, $G-e$ stands for an edge-deleted subgraph of the graph $G$. A similar situation, which holds in the case of the complete multipartite graph and its edge-deleted subgraph, is described by Proposition 3.9 However, two statements relevant for proving this proposition will be presented first. The reader who needs to be reminded of the adjacency spectrum of the complete multipartite graph is referred to [4, p. 73].

Theorem 3.7 (Interlacing Theorem for Complete Multipartite Graphs, [6, Theorem 1]). The $n-1$ negative eigenvalues $\lambda_{p-n+2}, \ldots, \lambda_{p}$ of a complete $n$-partite graph $K\left(p_{i}\right)=K_{p_{1}, p_{2}, \ldots, p_{n}}$, of order $p=\sum_{i=1}^{n} p_{i}$, with $n$ partition numbers $p_{i}$ and $t$ distinct partition numbers $\bar{p}_{i}$, satisfy the inequalities

$$
p_{1} \leq-\lambda_{p-n+2} \leq p_{2} \leq \lambda_{p-n+3} \leq p_{3} \leq \cdots \leq p_{n-1} \leq-\lambda_{p} \leq p_{n} .
$$

Moreover, for $t-1$ of the negative eigenvalues $\lambda_{i}^{\star}(i=2, \ldots, t)$, the strict inequalities

$$
\bar{p}_{1}<-\lambda_{2}^{\star}<\bar{p}_{2}<-\lambda_{3}^{\star}<\cdots<\bar{p}_{t-1}<-\lambda_{t}^{\star}<\bar{p}_{t}
$$

hold, and together with $\lambda_{1}$ they are the roots of the equation

$$
1-\sum_{i=1}^{t} \frac{n_{i} \bar{p}_{i}}{x+\bar{p}_{i}}=0 .
$$

Theorem 3.8 ([4, Theorem 6.7]). A graph has exactly one positive eigenvalue if and only if its non-isolated vertices form a complete multipartite graph. 
Proposition 3.9. Let $G=K_{p \times k}$ be the complete p-partite graph of order $n=p k$, and let $\lambda_{1}(G-e) \geq \lambda_{2}(G-e) \geq \cdots \geq \lambda_{n}(G-e)$ be the A-spectrum of the graph $G-e$ such that $\lambda_{2}(G-e)+\lambda_{n-p+2}(G-e)=1-k$. Then the following holds:

$$
\sigma_{A}(G, G-e)=\sigma_{L}(G, G-e)=\sigma_{Q}(G, G-e)=2 .
$$

Proof. The graph $G^{*}=K_{k-1}, \underbrace{k, k, \ldots, k}_{p-1}$, of order $n-1$, is an induced subgraph of

the graph $G-e$. According to Theorem 3.7 the $A$-eigenvalues of $G^{*}$ are $\lambda_{1}\left(G^{*}\right)$, $[0]^{n-1-p}, \lambda_{n-p+1}\left(G^{*}\right) \in[-k,-(k-1)]$ and $[-k]^{p-2}$. By using Corollary 3.4 it can be found that the $A$-eigenvalues of $G-e$ are $[0]^{n-p-2}$ and $[-k]^{p-3}$. For the greatest and the smallest eigenvalue, the following inequalities hold: $\lambda_{1}(G-e)>0$ and $\lambda_{n}(G-e) \leq-k$. Since $G-e$, according to Theorem 3.8 , is not a complete multipartite graph, $\lambda_{2}(G-e) \neq 0$, i.e. $\lambda_{2}(G-e) \in\left(0, \lambda_{1}\left(G^{*}\right)\right]$. Also, $\lambda_{n-p+1}(G-$ $e) \in\left[\lambda_{n-p+1}\left(G^{*}\right), 0\right]$ and $\lambda_{n-p+2}(G-e) \in\left[-k, \lambda_{n-p+1}\left(G^{*}\right)\right]$. Since the $A$-spectrum of $G$ is known, one can easily obtain:

$$
\begin{aligned}
& \sigma(G, G-e) \\
& =\lambda_{1}(G)-\lambda_{1}(G-e)+\lambda_{2}(G-e)-\lambda_{n-p+1}(G-e)+\lambda_{n-p+2}(G-e)-\lambda_{n}(G-e) \\
& =n-k-\left(k(p-3)-\lambda_{2}(G-e)-\lambda_{n-p+2}(G-e)\right)+\lambda_{2}(G-e)+\lambda_{n-p+2}(G-e) \\
& =2\left(k+\lambda_{2}(G-e)+\lambda_{n-p+2}(G-e)\right)=2 .
\end{aligned}
$$

The remaining two equalities follow from Proposition 3.2

Let $G$ be a graph with vertex set $V(G)$. The partition $V(G)=V_{1} \dot{\cup} V_{2} \dot{\cup} \cdots \dot{U} V_{k}$, where $\dot{U}$ stands for the disjoint union, is an equitable partition if every vertex in $V_{i}$ has the same number of neighbours in $V_{j}$, for all $i, j \in\{1,2, \ldots, k\}$ (for more details see e.g. [5, p. 83]).

The following statements, used in Remark 3.12 below, were proved in [5]

Corollary 3.10 ([5, Corollary 1.3.13]). Let $A$ be a real symmetric matrix with spectrum $\lambda_{1} \geq \lambda_{2} \geq \cdots \geq \lambda_{n}$. Given a partition $\{1,2, \ldots, n\}=\Delta_{1} \dot{\cup} \Delta_{2} \dot{\cup} \cdots \dot{\cup} \Delta_{m}$ with $\left|\Delta_{i}\right|=n_{i}>0$, consider the corresponding blocking $A=\left(A_{i j}\right)$, where $A_{i j}$ is an $n_{i} \times n_{j}$ block. Let $e_{i j}$ be the sum of the entries in $A_{i j}$ and set $B=\left(e_{i j} / n_{i}\right)$ (note that $e_{i j} / n_{i}$ is the average row sum in $\left.A_{i j}\right)$. Then the eigenvalues of $B$ interlace those of $A$.

Theorem 3.11 ([5, Theorem 1.3.14]). Let $A$ be any matrix partitioned into blocks, as in Corollary 3.10. Let us suppose that the block $A_{i j}$ has constant row sums $b_{i j}$, and let $B=\left(b_{i j}\right)$. Then the spectrum of $B$ is contained in the spectrum of $A$ (taking into account the multiplicities of the eigenvalues).

Remark 3.12. It can be checked that $\sigma_{A}\left(K_{n_{1}, n_{2}}, K_{n_{1}, n_{2}}-e\right) \neq 2$, for $n_{1} \neq n_{2}$. Namely, let $V\left(K_{n_{1}, n_{2}}\right)=X \cup Y$ be the set of vertices of the graph $K_{n_{1}, n_{2}}$, where $X=\left\{x_{i} \mid 1 \leq i \leq n_{1}\right\}, Y=\left\{y_{j} \mid 1 \leq j \leq n_{2}\right\}, n_{1}<n_{2}$, and let $e=x_{1} y_{1}$ be one of its edges. Then the partition $\left\{C_{1}, C_{2}, C_{3}, C_{4}\right\}$ of the set of vertices of $K_{n_{1}, n_{2}}-e$, where $C_{1}=\left\{x_{1}\right\}, C_{2}=\left\{x_{2}, \ldots, x_{n_{1}}\right\}, C_{3}=\left\{y_{1}\right\}$ and $C_{4}=\left\{y_{2}, \ldots, y_{n_{2}}\right\}$, is an 
equitable partition with the quotient matrix

$$
Q=\left(\begin{array}{cccc}
0 & 0 & 0 & n_{2}-1 \\
0 & 0 & 1 & n_{2}-1 \\
0 & n_{1}-1 & 0 & 0 \\
1 & n_{1}-1 & 0 & 0
\end{array}\right)
$$

The $i$-th row and column of $Q$ correspond to $C_{i}, i=1,2,3,4$. The characteristic polynomial of the matrix $Q$ is $q(x)=x^{4}-\left(n_{1} n_{2}-1\right) x^{2}+\left(n_{1}-1\right)\left(n_{2}-1\right)$, while its eigenvalues are

$$
x_{1,2}= \pm \frac{1}{\sqrt{2}} \sqrt{n_{1} n_{2}-1-\sqrt{\left(1-n_{1} n_{2}\right)^{2}-4\left(n_{1}-1\right)\left(n_{2}-1\right)}}
$$

and

$$
x_{3,4}= \pm \frac{1}{\sqrt{2}} \sqrt{n_{1} n_{2}-1+\sqrt{\left(1-n_{1} n_{2}\right)^{2}-4\left(n_{1}-1\right)\left(n_{2}-1\right)}} .
$$

According to Theorem 3.11, these eigenvalues are also the $A$-eigenvalues of the graph $K_{n_{1}, n_{2}}-e$. Since $K_{n_{1}, n_{2}-1}$ is an induced subgraph of $K_{n_{1}, n_{2}}-e$, from Corollary 3.4 it follows that in the $A$-spectrum of $K_{n_{1}, n_{2}}-e$ there are $n_{1}+n_{2}-4$ eigenvalues which are equal to 0 . Since

$$
\begin{gathered}
\sigma_{A}\left(K_{n_{1}, n_{2}}, K_{n_{1}, n_{2}}-e\right) \\
=2 \sqrt{n_{1} n_{2}}-\frac{2}{\sqrt{2}} \sqrt{n_{1} n_{2}-1+\sqrt{\left(1-n_{1} n_{2}\right)^{2}-4\left(n_{1}-1\right)\left(n_{2}-1\right)}} \\
+\frac{2}{\sqrt{2}} \sqrt{n_{1} n_{2}-1-\sqrt{\left(1-n_{1} n_{2}\right)^{2}-4\left(n_{1}-1\right)\left(n_{2}-1\right)}},
\end{gathered}
$$

it follows that $\sigma_{A}\left(K_{n_{1}, n_{2}}, K_{n_{1}, n_{2}}-e\right)=2$ only if $n_{1} \geq 1$ and $n_{1}=n_{2}$.

Proposition 3.13. For $n>2$ and $m \geq 2$, the following holds:

$$
\sigma_{A}\left(K_{n} \nabla K_{m, m},\left(K_{n}-e\right) \nabla K_{m, m}\right)=2 .
$$

Proof. By applying Theorem 1.2, one can easily obtain that the $A$-spectrum of $K_{n} \nabla K_{m, m}$ consists of the following eigenvalues: $x_{1},[0]^{2 m-2},[-1]^{n-1}, x_{2}$ and $[-m]$, where $x_{1,2}=\frac{1}{2}\left((n+m-1) \pm \sqrt{(n-1)^{2}+2 m(3 n+1)+m^{2}}\right)$.

The adjacency matrix $A\left(\left(K_{n}-e\right) \nabla K_{m, m}\right)$ of the graph $\left(K_{n}-e\right) \nabla K_{m, m}$ has the blocking as follows:

$$
A\left(\left(K_{n}-e\right) \nabla K_{m, m}\right)=\left(\begin{array}{ccc}
O & J & J \\
J & J-I & J \\
J & J & A\left(K_{m, m}\right)
\end{array}\right),
$$

where $J$ is the all-1 matrix, while $I$ is the identity matrix. Therefore, the quotient matrix $Q$ which corresponds to the matrix $A\left(\left(K_{n}-e\right) \nabla K_{m, m}\right)$ is

$$
Q=\left(\begin{array}{ccc}
0 & n-2 & 2 m \\
2 & n-3 & 2 m \\
2 & n-2 & m
\end{array}\right)
$$


while the characteristic polynomial of the matrix $Q$ is

$$
q(x)=-x^{3}+(n+m-3) x^{2}+(m n+2 n+3 m-4) x+2 n m .
$$

The roots $y_{1}, y_{2}, y_{3}$ of the polynomial $q(x)$ are eigenvalues of $\left(K_{n}-e\right) \nabla K_{m, m}$. The other eigenvalues of $\left(K_{n}-e\right) \nabla K_{m, m}$ remain the same if $J$ is subtracted from the blocks of $A\left(\left(K_{n}-e\right) \nabla K_{m, m}\right)$ which are equal to $J$, and from the block which is equal to $J-I$ (for more details related to this subject, see [3]). Thus, one obtains:

$$
A^{\prime}\left(\left(K_{n}-e\right) \nabla K_{m, m}\right)=\left(\begin{array}{ccc}
O & O & O \\
O & -I & O \\
O & O & A\left(K_{m, m}\right)
\end{array}\right) \quad \text { and } \quad Q^{\prime}=\left(\begin{array}{ccc}
0 & 0 & 0 \\
0 & -1 & 0 \\
0 & 0 & m
\end{array}\right) \text {. }
$$

The characteristic polynomial of the matrix $Q^{\prime}$ is $q^{\prime}(x)=(m-x) \cdot x \cdot(x+1)$, so the eigenvalues of $A^{\prime}$ which are not the eigenvalues of $Q^{\prime}$ are $[0]^{2 m-1},[-1]^{n-3}$ and $[-m]$. Therefore, the $A$-spectrum of the graph $\left(K_{n}-e\right) \nabla K_{m, m}$ consists of the eigenvalues $y_{1}, y_{2}, y_{3},[0]^{2 m-1},[-1]^{n-3}$ and $[-m]$.

In what follows, the order of the eigenvalues $y_{1}, y_{2}, y_{3}$ in the spectrum of the graph $\left(K_{n}-e\right) \nabla K_{m, m}$ will be determined. One of them, say $y_{1}$, is obviously the index of the graph. Since $K_{n-1} \nabla K_{m, m}$ is an induced subgraph of $\left(K_{n}-e\right) \nabla K_{m, m}$, according to Corollary 3.4 the remaining two eigenvalues, $y_{2}$ and $y_{3}$, may be in one of the following intervals (each of $y_{2}$ and $y_{3}$ in exactly one interval): $\left[0, x_{1}^{\prime}\right],[-1,0]$, $\left[x_{2}^{\prime},-1\right]$ and $\left[-m, x_{2}^{\prime}\right]$, where

$$
x_{1,2}^{\prime}=\frac{1}{2}\left(n-2+m \pm \sqrt{(n-2)^{2}+2 m(3 n-2)+m^{2}}\right)
$$

are the corresponding eigenvalues of the graph $K_{n-1} \nabla K_{m, m}$. Since the following relations hold: $q(0)=2 m n>0, q(-1)=(m-1)(n-2)>0, q(-m)=2 m\left(m^{2}-\right.$ $3 m+2)>0$,

$$
\begin{aligned}
& q\left(x_{2}^{\prime}\right)=\frac{1}{2}\left(2 m^{2}-6 m+5 m n-(n-2)^{2}\right) \\
&-\frac{1}{2}(2 m+n-2) \sqrt{m^{2}+(n-2)^{2}+m(6 n-4)}<0, \\
& q\left(x_{1}^{\prime}\right)=\frac{1}{2}\left(2 m^{2}+(n-2) \cdot\left(n-2+\sqrt{m^{2}+(n-2)^{2}+m(6 n-4)}\right)\right) \\
& \quad+\frac{m}{2}\left(5 n-6+2 \sqrt{m^{2}+(n-2)^{2}+m(6 n-4)}\right)>0,
\end{aligned}
$$

it can be concluded that $y_{2} \in\left[x_{2}^{\prime},-1\right]$ and $y_{3} \in\left[-m, x_{2}^{\prime}\right]$. More precisely, as

$$
q\left(x_{2}\right)=1-3 m-n+\sqrt{m^{2}+(n-1)^{2}+m(6 n+2)}<0
$$

and $q(-m)>0$, one obtains $y_{3} \in\left[-m, x_{2}\right]$.

So, one finally finds:

$\sigma_{A}\left(K_{n} \nabla K_{m, m},\left(K_{n}-e\right) \nabla K_{m, m}\right)=\left|x_{1}-y_{1}\right|+|0-(-1)|+\left|(-1)-y_{2}\right|+\left|x_{2}-y_{3}\right|=2$. 


\section{About the CONJeCture Related to the ADJACEnCy SPECTRAL DISTANCES OF CONNECTED REGULAR GRAPHS}

In [9], Z. Stanic posed the following six conjectures related to the $A$-spectral distances of graphs:

Conjecture 4.1. The A-spectral distance between any two graphs of order $n$ does not exceed $E_{n}^{\max }=\max \{E(G): G$ is a graph of order $n\}$, i.e.

$$
\operatorname{sdiam}^{A}\left(\mathcal{G}_{n}\right)=E_{n}^{\max }
$$

where $\mathcal{G}_{n}$ is the set of all n-vertex graphs.

Conjecture 4.2. Let $R_{1}$ and $R_{2}$ be the graphs having the maximum A-spectral distance among the connected regular graphs of order $n$. Then one of them is $K_{n}$, i.e.

$$
\operatorname{sdiam}^{A}\left(\mathcal{R}_{n}\right)=\operatorname{secc}_{\mathcal{R}_{n}}^{A}\left(K_{n}\right),
$$

where $\mathcal{R}_{n}$ is the set of all connected regular graphs of order $n$.

Conjecture 4.3. Let $B_{1}$ and $B_{2}$ be the graphs having the maximum A-spectral distance among the connected bipartite graphs of order $n$. Then one of them is $K_{\left\lceil\frac{n}{2}\right\rceil,\left\lfloor\frac{n}{2}\right\rfloor}$, i.e.

$$
\operatorname{sdiam}^{A}\left(\mathcal{B}_{n}\right)=\operatorname{secc}_{\mathcal{B}_{n}}^{A}\left(K_{\left\lceil\frac{n}{2}\right\rceil,\left\lfloor\frac{n}{2}\right\rfloor}\right),
$$

where $\mathcal{B}_{n}$ is the set of all connected bipartite graphs of order $n$.

Conjecture 4.4. The A-spectral distance between any two trees of order $n$ does not exceed $\sigma_{A}\left(P_{n}, K_{1, n-1}\right)$, i.e.

$$
\operatorname{sdiam}^{A}\left(\mathcal{T}_{n}\right)=\sigma_{A}\left(P_{n}, K_{1, n-1}\right),
$$

where $\mathcal{T}_{n}$ is the set of all trees of order $n$.

Conjecture 4.5. The following holds:

$$
\begin{gathered}
0.945 \approx \lim _{n \rightarrow+\infty} \sigma_{A}\left(P_{n}, Z_{n}\right)=\lim _{n \rightarrow+\infty} \sigma_{A}\left(W_{n}, Z_{n}\right)=\frac{1}{2} \lim _{n \rightarrow+\infty} \sigma_{A}\left(P_{n}, W_{n}\right), \\
\lim _{n \rightarrow+\infty} \sigma_{A}\left(C_{2 n}, Z_{2 n}\right)=\lim _{n \rightarrow+\infty} \sigma_{A}\left(C_{2 n-1}, Z_{2 n-1}\right)=2,
\end{gathered}
$$

where $Z_{n}$ and $W_{n}$ are, respectively, the snake graph and the double snake graph of order $n$.

Conjecture 4.6. For any $\epsilon>0$, there are graphs $G_{1}$ and $G_{2}$ having no common eigenvalues such that $\sigma_{A}\left(G_{1}, G_{2}\right)<\epsilon$.

Conjecture 4.1 was disproved in [8], and now Conjecture 4.2 will be considered.

Let $\mathcal{R}_{n}$ be the set of connected regular graphs of order $n$, and let $G \in \mathcal{R}_{n}$ be a $r$-regular graph whose adjacency spectrum is $r=\lambda_{1}(G) \geq \lambda_{2}(G) \geq \cdots \geq \lambda_{n}(G)$.

One can easily verify that

$$
\sigma_{A}\left(G, K_{n}\right)=|n-1-r|+\sum_{i=2}^{n}\left|-1-\lambda_{i}(G)\right|=E(\bar{G}),
$$

where $\bar{G}$ is the complement of $G$, and $\bar{G}$ is $(n-1-r)$-regular. Also, $\overline{\bar{G}}=G$. 
Since the energy $E(G)$ of a graph $G$ on $n$ vertices is not greater than $\frac{n}{2}(1+\sqrt{n})$ (see [11]), one can conclude that

$$
\operatorname{secc}_{\mathcal{R}_{n}}^{A}\left(K_{n}\right) \leq \frac{n}{2}(1+\sqrt{n}),
$$

which means, according to the statement given by Conjecture 4.2 that

$$
\operatorname{sdiam}^{A}\left(\mathcal{R}_{n}\right) \leq \frac{n}{2}(1+\sqrt{n}) .
$$

The generalized quadrangle $G=G Q(3,9)$ (for more details about this kind of graphs, see [7]) is the strongly regular graph with parameters $(112,30,2,10)$, whose $A$-spectrum is $30,[2]^{90},[-10]^{21}$. Since

$$
\sigma_{A}(G, \bar{G})=690>648,648 \approx \frac{112}{2}(1+\sqrt{112}) \geq \operatorname{secc}_{\mathcal{R}_{112}}^{A}\left(K_{112}\right),
$$

and since both graphs, $G=G Q(3,9)$ and its complement $\bar{G}$, are connected, Conjecture 4.2 is disproved.

\section{ACKNOWLEDGEMENT}

The author is grateful for the contributions of the reviewer, whose valuable and detailed comments led to an improved presentation of the results.

\section{REFERENCES}

[1] A. Abdollahi and M. R. Oboudi, Cospectrality of graphs, Linear Algebra Appl. 451 (2014), 169-181. MR 3198911

[2] A. Abdollahi, S. Janbaz and M. R. Oboudi, Distance between spectra of graphs, Linear Algebra Appl. 466 (2015), 401-408. MR 3278259

[3] S. M. Cioabă, W. H. Haemers, J. R. Vermette and W. Wong, The graphs with all but two eigenvalues equal to \pm 1 , J. Algebraic Combin. 41 (2015), no. 3, 887-897. MR 3328184

[4] D. M. Cvetković, M. Doob and H. Sachs, Spectra of Graphs, third edition, Johann Ambrosius Barth, Heidelberg, 1995. MR 1324340

[5] D. Cvetković, P. Rowlinson and S. Simić, An Introduction to the Theory of Graph Spectra, London Mathematical Society Student Texts, 75, Cambridge University Press, Cambridge, 2010. MR 2571608

[6] F. Esser and F. Harary, On the spectrum of a complete multipartite graph, European J. Combin. 1 (1980), no. 3, 211-218. MR 0593991

[7] C. Godsil and G. Royle, Algebraic Graph Theory, Graduate Texts in Mathematics, 207, Springer-Verlag, New York, 2001. MR 1829620.

[8] I. M. Jovanović, Some results on spectral distances of graphs, Rev. Un. Mat. Argentina 56 (2015), no. 2, 95-117. MR 3431817.

[9] I. Jovanović and Z. Stanić, Spectral distances of graphs, Linear Algebra Appl. 436 (2012), no. 5, 1425-1435. MR 2890928

[10] I. Jovanović and Z. Stanić, Spectral distances of graphs based on their different matrix representations, Filomat 28 (2014), no. 4, 723-734. MR 3360065

[11] J. H. Koolen and V. Moulton, Maximal energy graphs, Adv. in Appl. Math. 26 (2001), no. 1, 47-52. MR 1806691. 
[12] Q. Li and K. Q. Feng, On the largest eigenvalue of a graph, Acta Math. Appl. Sinica 2 (1979), no. 2, 167-175. MR 0549045

[13] R. Liu, H. Jia and J. Shu, An edge-rotating theorem on the least eigenvalue of graphs, Acta Math. Appl. Sin. Engl. Ser. 31 (2015), no. 4, 945-952. MR 3418272

[14] D. Stevanović, Research problems from the Aveiro Workshop on Graph Spectra, Linear Algebra Appl. 423 (2007), no. 1, 172-181. MR 2312333

Irena M. Jovanović

School of Computing, Union University, Knez Mihailova 6, 11000 Belgrade, Serbia

irenaire@gmail.com

Received: September 8, 2019

Accepted: August 26, 2020 